\title{
ESTUDO MORFODINÂMICO DURANTE UMA MARÉ EQUINOCIAL DE SIZÍGIA EM UMA PRAIA DE MACROMARÉ DO LITORAL AMAZÔNICO (PRAIA DE AJURUTEUA-PA, BRASIL)
}

\author{
MORPHODYNAMIC STUDY DURING AN EQUINOCTIAL SPRING TIDE IN \\ A MACROTIDAL BEACH OF THE AMAZON LITTORAL \\ (AJURUTEUA BEACH, PARÁ, BRAZIL)
}

\author{
Vitalina Magalhães Barbosa ${ }^{1}$ \\ Anderson Manoel da Silva Gregório ${ }^{1}$ \\ Débora Vieira Busman ${ }^{1}$ \\ Rauquírio André Albuquerque Marinho da Costa ${ }^{2}$ \\ Pedro Walfir Martins Sousa e Filho ${ }^{3}$ \\ Luci Cajueiro Carneiro Pereira ${ }^{1}$
}

\begin{abstract}
RESUMO
A zona costeira bragantina está susceptível a fortes processos hidrodinâmicos que continuamente remodelam a morfologia praial local. Estas flutuações de energia são mais intensas durante as marés equinociais de sizígia, registradas durante os meses de março/abril e setembro/outubro, na região. Para conhecer as variações morfodinâmicas durante um destes períodos, medidas de intensidade e direção de correntes, altura da onda na rebentação $\left(H_{b}\right)$, período da onda $(T)$, ângulo de rebentação $\left(a_{b}\right)$, declividade do perfil $(m)$, surf scaling parameter $(\varepsilon)$, coeficiente de rebentação $(\beta)$ e variação da escarpa, foram realizadas, diariamente, entre os dias 17 e 22 de março de 2003. As correntes foram medidas na zona de surf, através de um correntômetro Sensordata SD30. As alturas, períodos e ângulo de rebentação foram medidos com o auxílio de régua, cronômetro e bússola, respectivamente. Os levantamentos topográficos foram realizados em dois perfis perpendiculares à linha de costa, com auxílio de um nível, tripé e mira falante. As análises realizadas demonstraram que a área
\end{abstract}

1 Laboratório de Oceanografia Costeira e Estuarina, Instituto de Estudos Costeiros, Campus Universitário de Bragança-UFPA, Alameda Leandro Ribeiro, s/n, Bragança, Pará, Brazil. CEP: 68600-000. E-mail:cajueiro@ufpa.br. Fone: 559124251209.

2 Laboratório de Plâncton e cultivo de Microalgas, Campus Universitário de Bragança, Universidade Federal do Pará, Aldeia, CEP: 68600-000, Bragança, Pará, Brasil. Fone: 5591 3425-4536.

3 Laboratório de Análise de Imagens do Trópico Úmido (LAIT), Centro de Geociências, Universidade Federal do Pará, Av. Augusto Correa 1, Guamá, CEP: 66075-110, C.P. 8608, Belém, Pará, Brasil. Fone: 5591 3201-8009. 
BARBOSA, V. M. et al. Estudo morfodinâmico durante uma maré equinocial de Sizígia ...

em estudo é, em sua maioria, morfodinâmicamente dissipativa, com ondas tipo deslizante principalmente, no setor SE. Os processos erosivos na praia em estudo são conseqüência de causas naturais, intensificadas por ações antrópicas (e.g. ocupação desordenada de edificações sobre campos de dunas e área de manguezal), sendo a energia hidrodinâmica gerada pela macromaré equinocial, um fator preponderante na modificação da configuração morfológica da praia de Ajuruteua, durante o período em estudo. Estas alterações refletiram em transformações morfodinâmicas a curto período que corresponderam a alterações no volume sedimentar dos diferentes subambientes praiais.

Palavras-chave: Morfodinâmica; maré equinocial; macromaré; litoral amazônico.

\section{ABSTRACT}

The Bragantinian coastal zone has been subjected to strong hydrodynamic processes, which continuously remodel its beach morphology. These energy fluctuations are more intense during the equinoctial spring tides, registered during the months of March/April and September/October. To understand the morphodynamic changes during an equinoctial spring tide event (from 17 to 22 of March of 2003), morfodynamic studies were carried out daily to characterize parameters such as current intensity and speed, wave break height $\left(\mathrm{H}_{\mathrm{b}}\right)$, wave break period $(T)$, wave break angle $\left(a_{b}\right)$, profile's declivity $(m)$, surf scaling parameter $(\varepsilon)$, breaking coefficient $(\beta)$ - and beach scarp variations. The currents were measured in the surf zone through a Sensordata SD30 current meter. The wave break height, period and angle were measured by a graduate rule, chronometer and manual compassing, respectively. The topographic surveys were carried out in two perpendicular shoreline profiles by role and topographic level. The analysis of these parameters showed that the studied area is, in its majority, dissipative with a spilling type breaker, mainly in the SE sector. The observed erosive processes in the studied beach are a consequence of natural causes (sometimes unknown) intensified by the human influence e.g. disordered occupation on dune areas and mangrove zones by edifices. The equinoctial macrotide energy was the principal factor that contributed to the appearance of morphodynamic modifications at the Ajuruteua beach. These variations were responsible for short-term sedimentary volume changes in the different beach sub-environments.

Key words: Morphodynamic; equinoctial tide; macrotidal; Amazon coast.

\section{INTRODUÇÃO}

O estudo da dinâmica da zona costeira é de suma importância, uma vez que nesta região, considerada altamente vulnerável às modificações antrópicas e/ou naturais, interagem processos marinhos, continentais e atmosféricos (Micallef \& Williams 2002, Pereira et al. 2003, Maia et al. 2005, entre outros).

Entre os ambientes costeiros, o sistema praial destaca-se por representar um importante elemento de proteção costeira, ao mesmo tempo em que são amplamente utilizados para o turismo e lazer (Nelson et al. 2000, Burak et al. 2004, Anfuso \& Gracia 2005, entre outros).

Apesar da existência de inúmeras definições sobre o termo praia, as mais recentes buscam nos processos hidrodinâmicos atuantes sobre a costa, a delimitação deste ambiente. Neste contexto, Horikawa (1988) considerou praia arenosa aquela região de sedimentos inconsolidados, situada na região costeira, e facilmente modificada por processos meteorológicos e oceanográficos.

O estado morfodinâmico praial depende de uma série de agentes interdependentes que condicionam o "equilíbrio morfológico-dinâmico" do sistema praial. As marés, correntes, ondas e ventos são agentes responsáveis pelo deslocamento dos sedimentos e pelo modelamento fisiográfico da costa, produzindo formas e feições erosivas e deposicionais bastante variáveis (Masselink et al. 2006, Lafon et al. 2005, Grunnet et al. 2005, Jackson et al. 2005, entre outros).

Inserida na maior área contínua de manguezal do mundo (Kjerfve et al. 2002), a costa paraense apresenta configuração extremamente recortada e está submetida a processos hidrodinâmicos e meteorológicos bastante singulares e ainda pouco conhecidos, do ponto de vista científico.

O regime de macromarés semi-diurnas na região induz a formação das correntes de marés e exerce um importante papel na circulação local, influenciando fortemente o transporte sedimentar litorâneo (Souza Filho \& Paradella 2002, Souza Filho \& Paradella 2003), constituindo, um fator bastante importante nas variações morfológicas da linha de costa, principalmente, durante as marés equinociais de sizígia.

No nordeste paraense está situada a Planície Costeira Bragantina que, por sua vez, encontra-se inserida na bacia Bragança-Viseu, a qual possui um regime neotectônico distensivo e, juntamente com as flutuações do nível do mar, vem controlando a evolução da paisagem desta região (Souza Filho et al. 2006). 
BARBOSA, V. M. et al. Estudo morfodinâmico durante uma maré equinocial de Sizígia ...

Neste contexto, situa-se a praia de Ajuruteua, localizada na faixa costeira do município de Bragança, entre o canal da Barca e o canal de Chavascal.

A praia de Ajuruteua possui, cerca de $2,5 \mathrm{~km}$ de extensão, na qual a ocupação ocorre, principalmente, nos primeiros $500 \mathrm{~m}$ acima da linha de preamar de sizígia. Com cerca de 400 moradores permanentes (maioria pescadores e/ou comerciantes), esta praia é uma das mais freqüentadas por veranistas do litoral amazônico, entretanto o aumento do nível do mar, o ambiente de alta energia (em decorrência das macromarés) e a ocupação desordenada das edificações locais (casas, bares e pousadas) são fatores que contribuem com o estado erosivo que se encontra o setor NW da praia em questão (Souza Filho et al. 2003 e Pereira et al. In press). $\mathrm{O}$ avanço do mar, além de destruir as edificações mais próximas à costa, vem afetando também, outros ambientes associados e.g. manguezal, restinga e dunas, comprometendo entre outros, a produtividade e diversidade local, o turismo na região, assim como as atividades socioeconômicas na vila de Ajuruteua (Pereira et al. In press).

Visando entender melhor a dinâmica da praia de Ajuruteua, um monitoramento sobre a morfologia praial e a hidrodinâmica costeira local foi realizado durante um evento de curto período, conhecido como maré equinocial de sizígia.

\section{ÁREA DE ESTUDO}

A Costa Atlântica do Salgado Paraense, localizada na Mesorregião Nordeste Paraense, é tectonicamente do tipo trainling edge coast (Inman \& Filloux 1960), apresentando topografia plana e ampla plataforma continental, da qual faz parte a Microrregião Bragantina (Figura 1).

Inserida nesta microrregião, a Planície Costeira de Bragança apresenta cerca de $40 \mathrm{~km}$ de linha de costa, estendendo-se desde a Ponta do Maiaú até a foz do Rio Caeté, entre as coordenadas $00^{\circ} 43^{\prime} 18^{\prime \prime}$ - $00^{\circ} 04^{\prime} 17^{\prime \prime} \mathrm{S}$ e 4632'16" - 4655'11' W. Esta planície está inserida na Bacia Costeira de Bragança-Viseu e sua geometria e paleotopografia estão associadas às movimentações tectônicas que têm controlado as espessuras dos depósitos terciários e quaternários (Souza Filho \& ElRobrini 1998).

Esta planície é caracterizada por uma dinâmica significativa, na qual seus ambientes sofrem transformações temporais de curtos, médios e longos períodos, sobretudo, por tratar-se de uma costa transgressiva, dominada por macromarés semidiurnas, além de apresentar problemas relacionados à ocupação desordenada (Praia de Ajuruteua e Vila dos Pescadores), o que vêm afetando suas praias, dunas e manguezais (Krause et al. 2000, Krause \& Glaser 2003, Souza Filho et al. 2003, Pereira et al. In press, Souza Filho et al. 2006).

Neste contexto, situa-se a praia de Ajuruteua, também chamada de Campo do Meio, que margeia os manguezais adjacentes e encontra-se limitada pelos canais de marés, proporcionando um traçado retilíneo orientado, segundo a direção NW-SE, apresentando suas extremidades encurvadas (Souza Filho \& El Robrini 1997).

A praia de Ajuruteua apresenta uma extensa área plana de, aproximadamente, $2,5 \mathrm{~km}$ de extensão, que vem enfrentando um progressivo recuo na linha de costa, decorrente dos processos erosivos que predominam na região (Souza Filho et al. 2003). Por outro lado, apresenta 200-400 m de largura (medida de baixa-mar de sizígia) e suave inclinação em direção ao mar, estando classificada como uma barrier-beach ridge (Souza Filho \& El Robrini 1997).

O clima é equatorial úmido do tipo Amw', segundo o sistema KÖPPEN de classificação, e está caracterizado por uma estação muito chuvosa entre os meses de dezembro e maio e uma estação seca, nos demais meses do ano. A pluviosidade anual varia de 2500 a $3000 \mathrm{~mm}$, enquanto a umidade relativa está entre $80 \mathrm{e}$ $91 \%$. A temperatura média anual é, aproximadamente, de $25,7^{\circ} \mathrm{C}$ variando ao longo do ano de $20,4^{\circ} \mathrm{C}$ a $32,8^{\circ} \mathrm{C}$ (Martorano et al. 1993).

$\mathrm{Na}$ região predominam os ventos alísios de NE, com velocidades médias de $7,9 \mathrm{~m} / \mathrm{s}$, principalmente, entre os meses de dezembro e maio. Esses ventos são, geralmente, precedidos de calmaria e, quase sempre, acompanhados de rajadas violentas e chuvas intensas. Entre os meses de junho e novembro, predominam os ventos $E$ e SE, com intensidades moderadas (Silva 2001).

Nesta área predominam as macromarés semidiurnas, com altura média de 4,8 m e alturas máximas superiores a $5,5 \mathrm{~m}$, durante as marés equinociais de sizígia (DHN 2003).

As principais correntes que atuam no litoral paraense são as correntes de maré e, secundariamente, as correntes litorâneas resultantes da chegada das ondas na costa, que são responsáveis pelo transporte de sedimentos da plataforma continental para o litoral (Alves 2001).

A cobertura vegetal desta região é caracterizada por um denso bosque de mangue e uma grande diversidade de ambientes sedimentares que incluem planícies de marés, pântanos salinos, cheniers, campos de dunas 
e outros (Souza Filho \& Paradella 2003).

A vegetação de mangue ocupa toda a Planície Costeira de Bragança, penetrando em direção ao continente ao longo dos canais estuarinos. Esta vegetação ocorre nas áreas com influência de águas salinas e salobras, sendo constituídas somente por comunidades típicas, como Rhizophora, Avicennia e Laguncularia (Lara 2003 e Krause \& Glaser 2003).

\section{MATERIAIS E MÉTODOS}

Os estudos foram realizados na praia de Ajuruteua, durante os dias 17 e 22 de março de 2003, durante uma maré equinocial de sizígia de lua cheia, em duas estações (E1 e E2, Figura 1). Os estudos incluíram o levantamento topográfico de perfis praiais, distância das escarpas, medidas de correntes, alturas de ondas na rebentação $\left(\mathrm{H}_{\mathrm{b}}\right)$, período de ondas $(T)$ e ângulo de incidência $\left(a_{b}\right)$. Com os valores obtidos foi possível determinar a declividade do perfil $(\mathrm{m})$, o surf scaling parameter $(\varepsilon)$ e o coeficiente de rebentação da onda.

\section{Morfologia Praial}

Para determinar a morfologia praial, dois perfis topográficos (Pp1 e Pp2) foram monitorados, diariamente, durante o período de 17 a 22/03.

O levantamento topográfico foi efetuado com o auxílio de um nível automático, que consta de uma mira apoiada em um tripé e uma régua escalonável de $5 \mathrm{~m}$. Os dois transects foram monitorados a partir de um referencial fixo (E0), situados nos campos de dunas, até o limite superior da antepraia. A metodologia empregada foi baseada no método topográfico da Stadia, aperfeiçoado para ambiente de praia por Birkemeier (1981).

Paralelamente, medidas de escarpa foram realizadas entre os dois perfis, em 10 pontos (Pe1 a Pe10), com

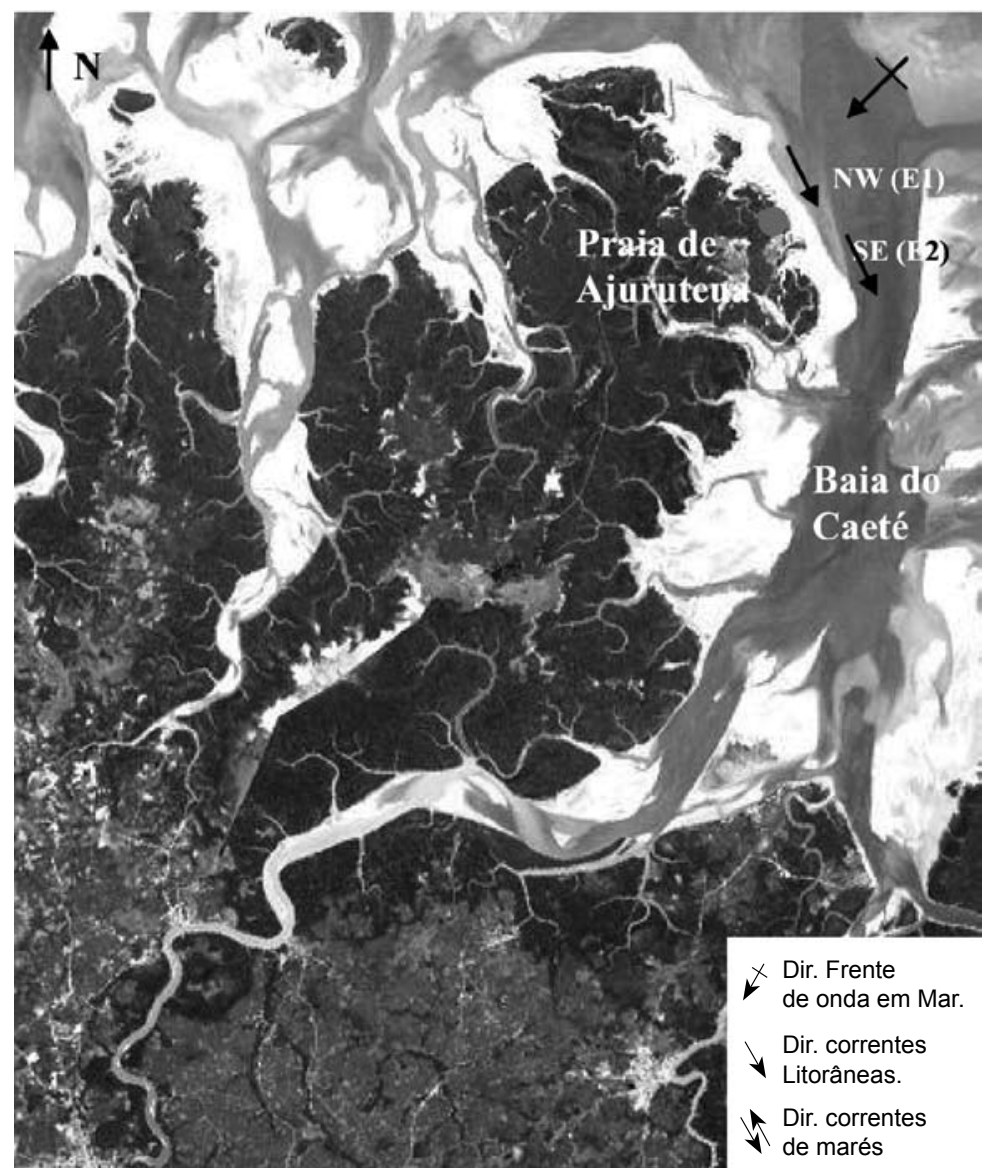

Figura 1 - Localização da área de estudo 
BARBOSA, V. M. et al. Estudo morfodinâmico durante uma maré equinocial de Sizígia ...

o auxílio de uma trena, a partir de pontos de referência (E0) situados nos campos de dunas.

\section{Hidrodinâmica Praial}

Os dados climatológicos (ventos e precipitação) foram obtidos por uma estação meteorológica da UFPA (Projeto LBA), situada próxima à praia de Ajuruteua. Os dados de ventos foram registrados a $28 \mathrm{~m}$ de altura, acima da copa da vegetação de mangue.

As intensidades das correntes costeiras foram medidas em dois pontos Pc1 e Pc2, nas imediações dos perfis praias, durante as marés enchentes e vazantes (20 a 22/03), na zona de surf. Cada leitura foi realizada após cinco minutos de imersão do correntômetro (Sensordata - SD30). Paralelamente, próximo ao Pc1, foram medidos as alturas significativa da onda na zona de rebentação, os períodos da onda e os ângulos de rebentação da onda, com o auxílio de uma régua graduada e um cronômetro, respectivamente, segundo metodologia adotada por Bascom (1964).

Para determinar o estado morfodinâmico da área em estudo foi utilizado o parâmetro surf scaling parameter, proposto por Guza \& Inman (1975) e aprimorado por Wright \& Short (1984). O tipo de rebentação de onda foi classificado, de acordo com a aplicação do coeficiente de rebentação proposto por Galvin (1968).

\section{RESULTADOS E DISCUSSÃO}

As diferentes respostas morfodinâmicas observadas entre os perfis estudados estão relacionadas, provavelmente, aos diferentes ambientes de energia, aos quais a praia de Ajuruteua está submetida. A forma de arco alongado na direção NW-SE e a predominância de ondas na direção NE são os principais responsáveis para que o setor NW de Ajuruteua seja considerado de alta energia, quando comparado com o setor SE, local em que as ondas são refratadas ao atingir a zona de rebentação.

\section{Morfologia Praial}

De acordo com Souza-Filho \& El-Robrini (2000), a praia de Ajuruteua pode ser classificada como uma barrier-beach ridges, subdividida em três zonas (i) póspraia, constituída por dunas cobertas por vegetação e berma; (ii) estirâncio, subdividido em três subzonas que dependem das alturas de marés (sizígia e quadratura) e (iii) antepraia, que representa a zona mais baixa, destas três zonas.

No setor SE (baixa a moderada energia) foi constatada a presença de barras arenosas e calhas, na zona de estirâncio, característica típica de uma praia de macromaré de estado dissipativo, acordando com Short (1982), Short (1991) e Short (1999). Souza Filho et al. (2003), após monitoramento da linha de praia, registraram que este trecho vem sendo acrescido, cerca de, 1,46 m/mês. Entretanto, durante este evento de curto período (ressaca) a praia perdeu $-0,14 \mathrm{~m}^{3} / \mathrm{m}$, ao longo do perfil. A erosão ocorreu, principalmente, na escarpa e na segunda barra longitudinal, enquanto as zonas superior e inferior do estirâncio sofreram acresção (Figura 2).

Os dados obtidos neste setor apontam que a praia se estendeu desde o nível de maré baixa de uma maré equinocial de sizígia a duna frontal, perfazendo um total de 370 metros. Durante este período, as dunas apresentaram-se escarpadas e o estirâncio com mais de 250 metros de extensão, nos quais foi possível observar duas barras arenosas e uma calha.

Por outro lado, o perfil P2, localizado no setor NW (moderada a alta energia), não apresentou o sistema barra-calha, conferindo a este perfil características erosivas e típicas de praia intermediária. Neste setor, foi possível observar ainda que os sedimentos foram erodidos, principalmente, da escarpa e depositados ao longo do estirâncio (Figura 2) e que houve erosão de $-0,22 \mathrm{~m}^{3} / \mathrm{m}$, durante o período em monitoramento. Estudos realizados por Souza Filho et al. em 2003 apontam que de fato existe uma tendência erosiva no setor NW e que cerca de -2,21 m de praia são erodidas por mês. Neste setor foi observado ainda, que a praia se estendeu desde o nível de maré baixa de sizígia até as dunas escarpadas, perfazendo cerca de 250 metros, dos quais, aproximadamente, $200 \mathrm{~m}$ foi de estirâncio.

Entre os pontos de monitoramento de variação de escarpa foi observado que aqueles localizados mais próximos ao setor erosivo Pp2 (Pe8, Pe9 e Pe10) apresentaram uma maior variação (erosão), quando comparados aos pontos localizados mais próximos ao setor acrescido Pp1 (p1, p2 e p3). A maior variação registrada foi de $5,9 \mathrm{~m}$, no Pe9 (setor NW) e a menor de 0,6 m, no Pe1 (setor SE) (Figura 3).

Com relação ao uso e ocupação existiu e vem 
existindo desmatamento de manguezal e de campos alagados para a ocupação de edificações. A ocupação desordenada sobre dunas também ocorre nos dois setores, e vem intensificando o processo erosivo, no setor NW. Neste setor, muitas edificações (bares, casas e pousadas) já foram destruídas ou desmontadas e transportadas para locais mais protegidos da influência direta da dinâmica costeira local (Figuras 4 e 5).

Estudos realizados por Pereira et al. (In press), entre 2003 e 2004, mostram que mais de 15 casas (no setor NW) foram destruídas ou transferidas para áreas mais protegidas da vila de Ajuruteua. Este deslocamento de estabelecimentos só é possível porque as casas são, na grande maioria (85\%), de madeira (Pereira et al. In press).

\section{HIDRODINÂMICA COSTEIRA}

A escassez de registros sobre os parâmetros oceanográficos, principalmente, sobre o regime de correntes e ondas, é um fator limitante na caracterização do regime hidrodinâmico atuante na região costeira paraense, fato que dificulta, ou mesmo, impossibilita uma análise quantitativa mais precisa sobre o tema. Abaixo seguem os resultados obtidos durante um evento de curto período (maré equinocial de sizígia).

\section{Climatologia}

Durante o mês de março predominaram os ventos $\mathrm{NE}$, com velocidade média de $1,9 \mathrm{~m} / \mathrm{s}$. Comparando este mês com os demais meses do ano de 2003 foi possível observar que em março foram registradas as menores intensidades de vento. Por outro lado, foi o mês que mais

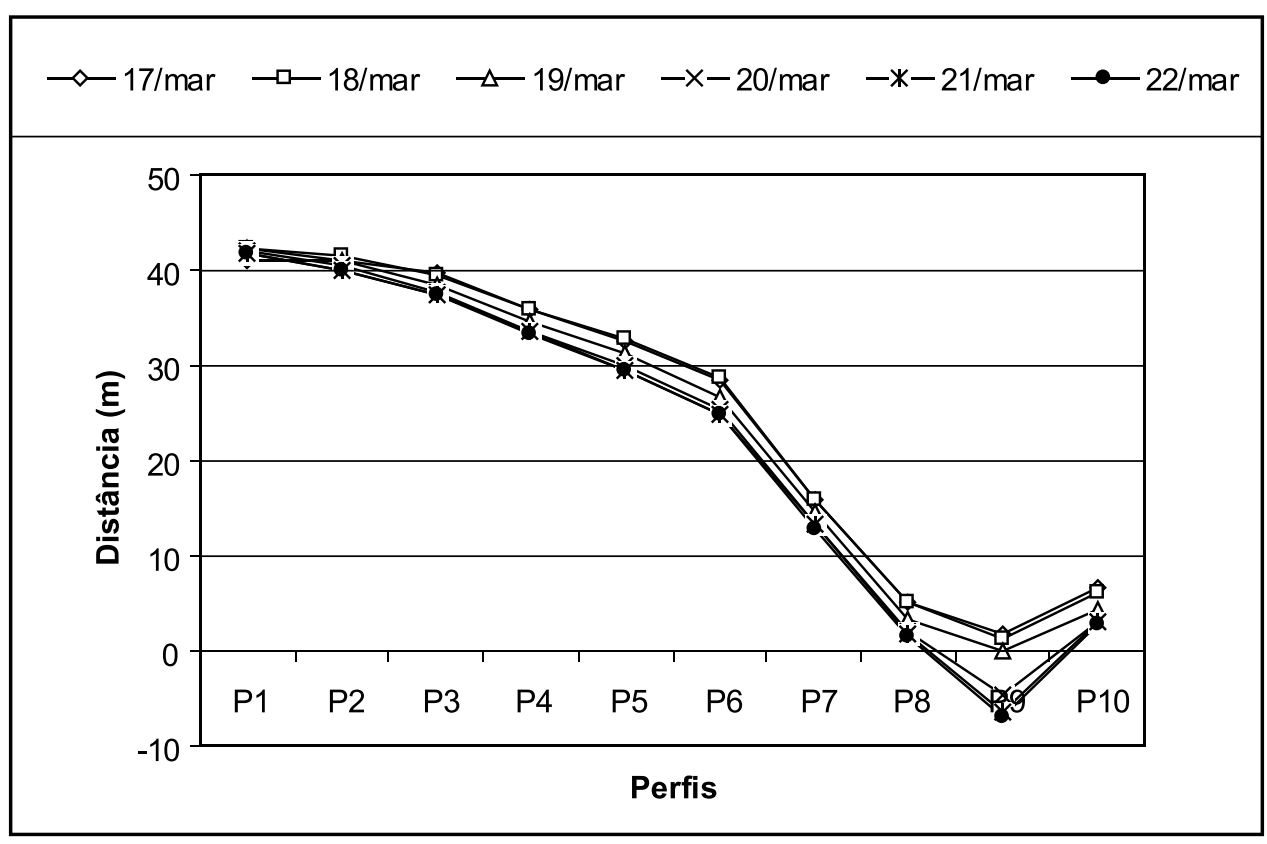

Figura 2 - Variação da escarpa, durante os dias 17 a 22/03/2003, em Ajuruteua-PA 


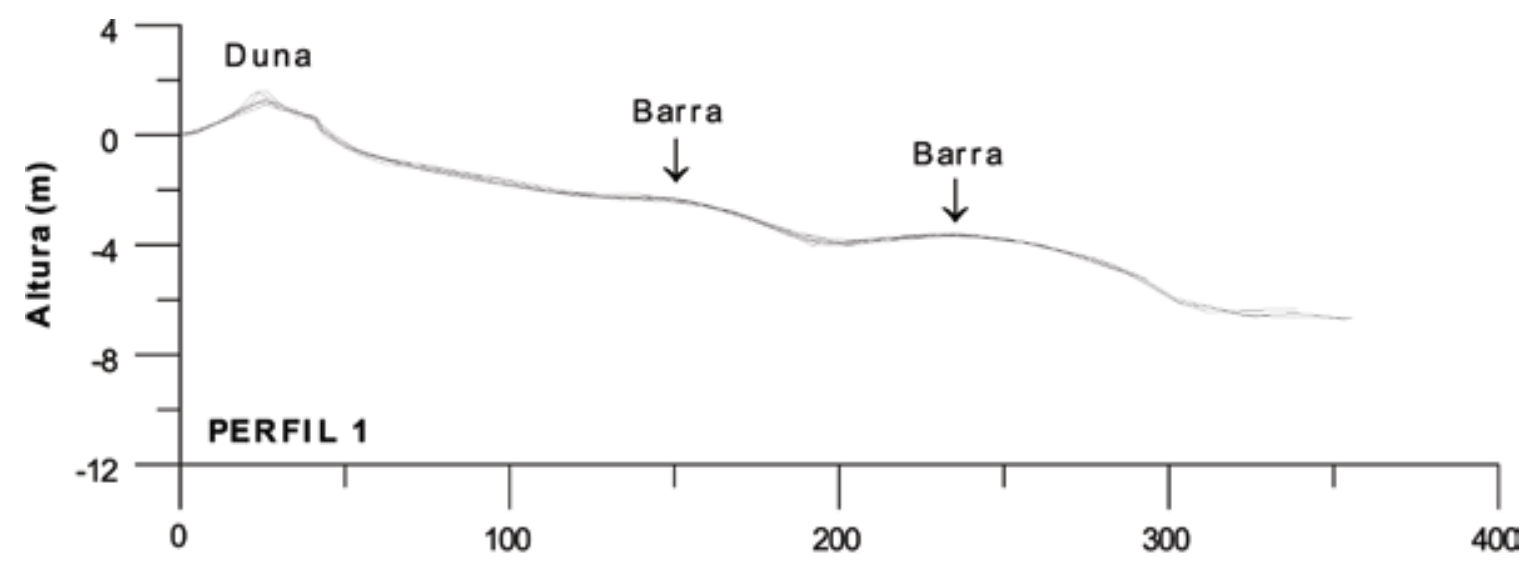

Distância (m)

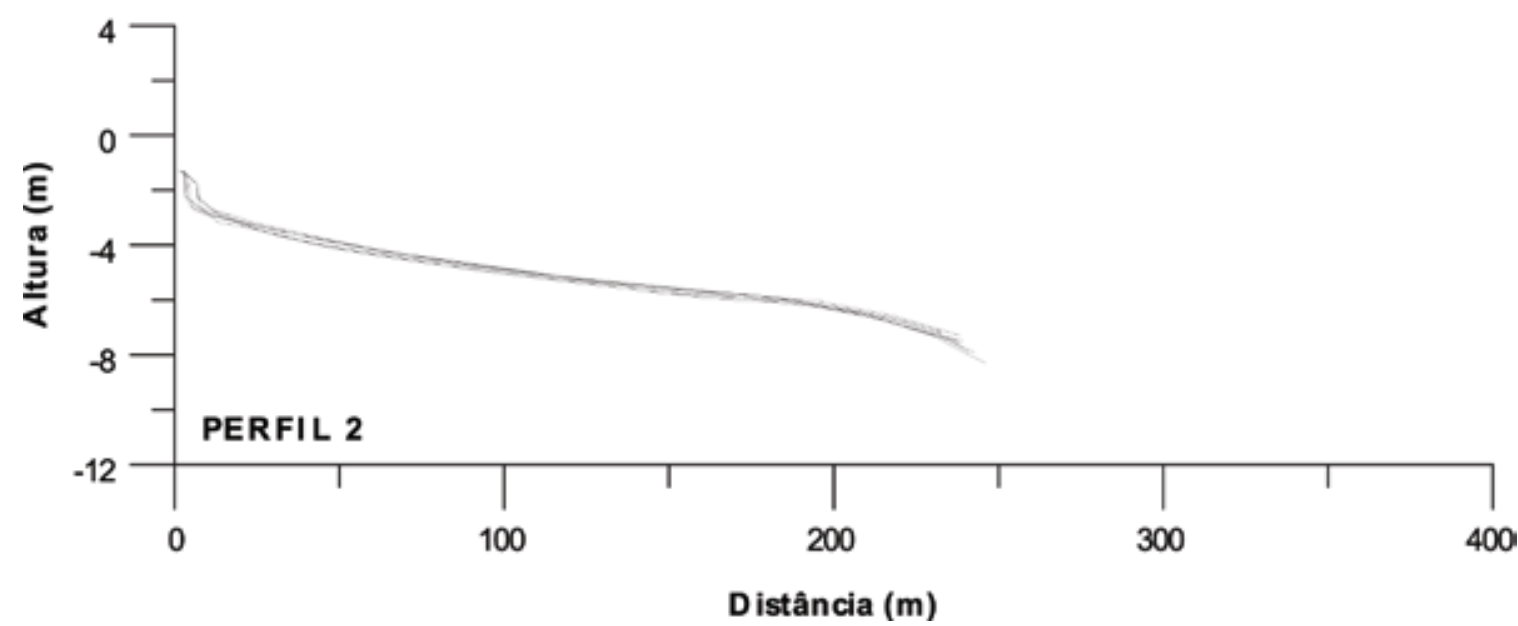

Figura 3 - Morfologia do Perfil 1 (Pp1, setor SE) e Perfil 2 (Pp2, setor NW), Ajuruteua-PA. NMPES (Nível médio de preamar equinocial de sizígia) e NMBES (Nivel médio de baixamar equinocial de sizígia)

choveu (precipitação superior a $800 \mathrm{~mm}$ ). Os dados de intensidade do vento e precipitação pluviométrica, do ano de 2003, estão representados pelas Figuras 6 e 7.

\section{Correntes}

As intensidades das correntes, na zona de surf, variaram de $10 \mathrm{~cm} / \mathrm{s}$ a $78 \mathrm{~cm} / \mathrm{s}$ (Figura 8 ), sendo os maiores valores registrados, principalmente no período de enchente, possivelmente em decorrência da direção predominante dos ventos vindos de NE. Por outro lado, a direção das correntes de maré foi NWSE durante os períodos de enchentes e SE-NW durante as vazantes. 


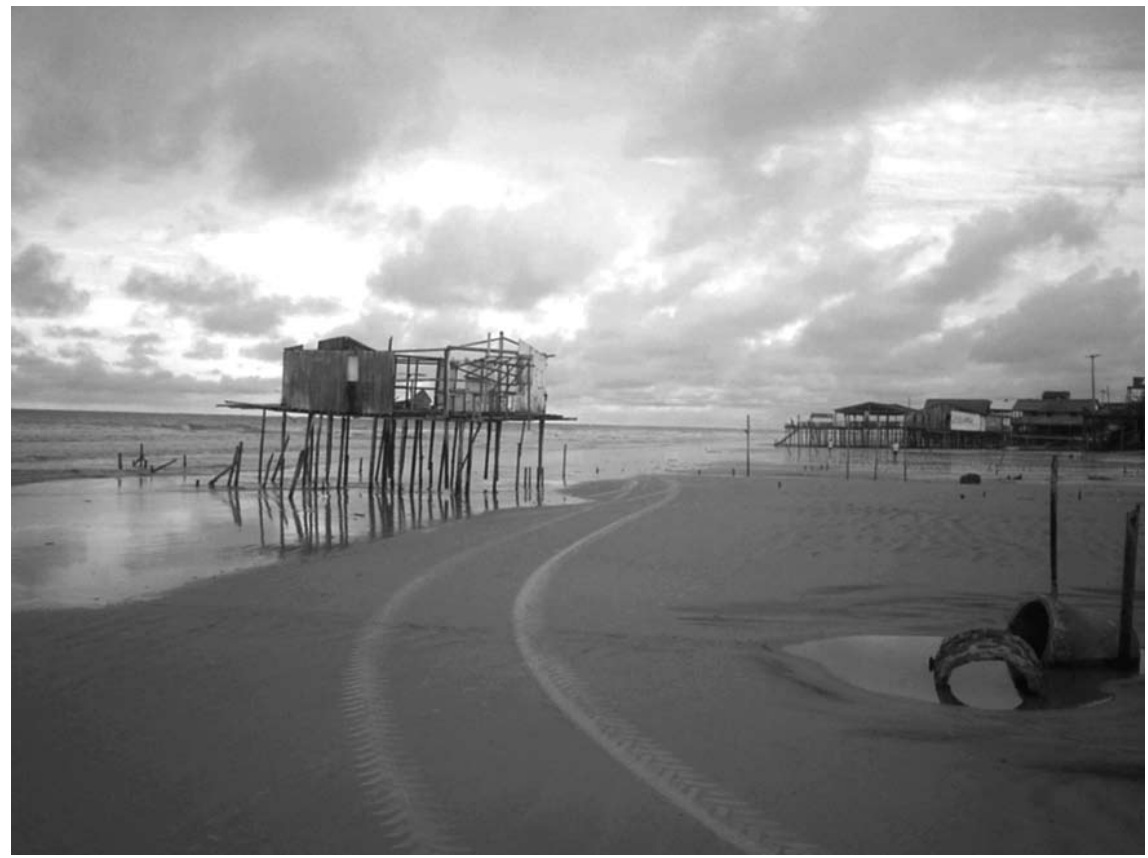

Figura 4 - Destruição de edificações no setor NW da praia de Ajuruteua-PA. Foto: Danielly Guimarães (2004)

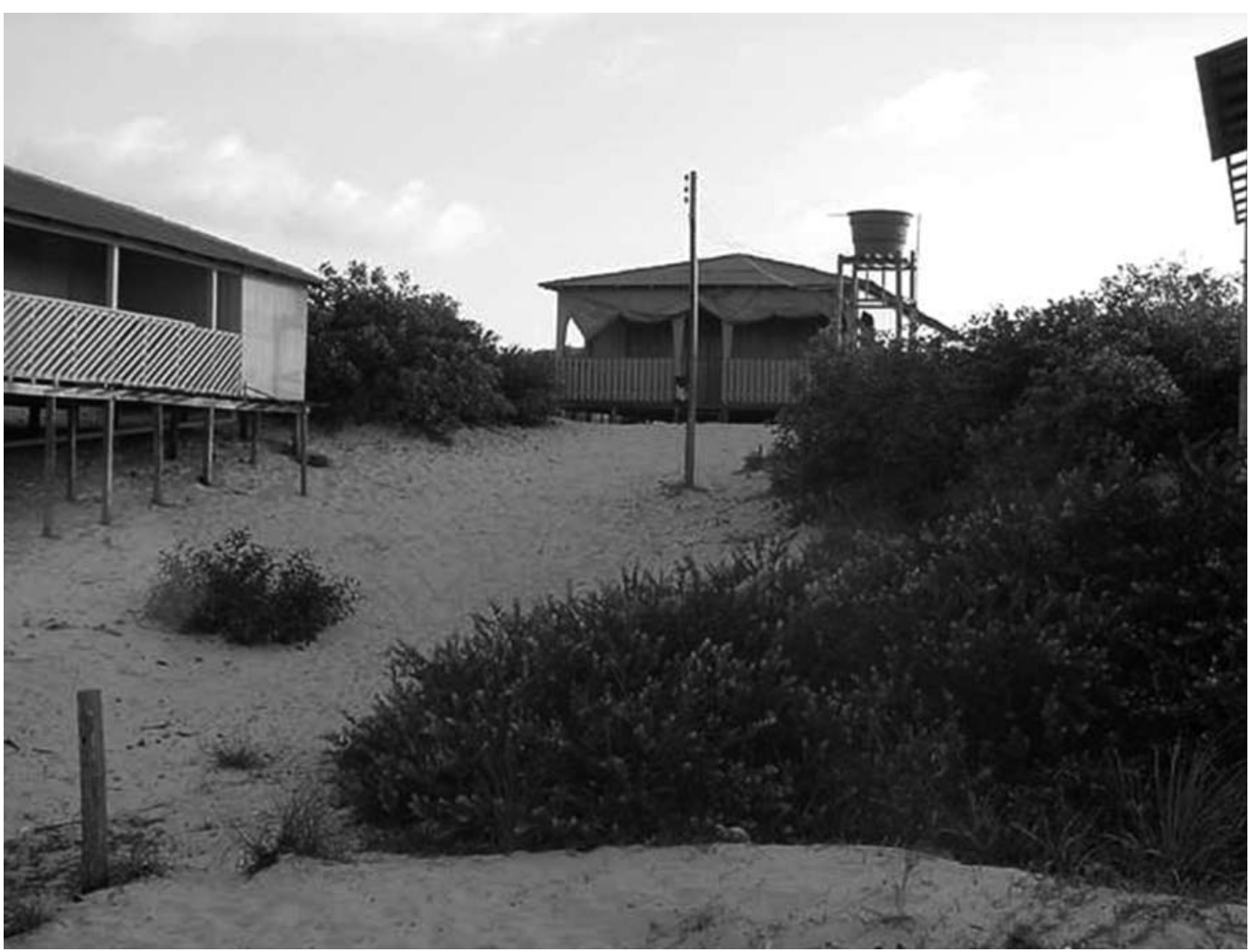

Figura 5 - Ocupação de edificações no setor SE da praia de Ajuruteua-PA. Foto: Danielly Guimarães (2004) 


\section{Ondas}

Entre os dias 20 e 22 de março de 2003, o ângulo predominante de rebentação de onda $\left(a_{b}\right)$ foi de $8^{\circ}$. $O$ valor médio da altura de onda na rebentação $\left(\mathrm{H}_{\mathrm{b}}\right)$ foi $0,8 \mathrm{~m}$ e o valor médio de $\mathrm{H}_{\mathrm{b}} 1 / 3$ foi $1,2 \mathrm{~m}$, para os períodos de $8,8 \mathrm{~s}$ e $9,2 \mathrm{~s}$, respectivamente.

\section{Estado Morfodinâmico}

De acordo com o modelo sugerido por Guza \&
Inman (1975) e Wright \& Short (1984), as ondas da praia de Ajuruteua, apresentaram valores de estados morfodinâmicos $(\epsilon)$, superiores a 20 , indicando o estado dissipativo com ondas tipo deslizante para os dois perfis.

Entre os perfis estudados, o Pp1 foi o que apresentou o estado mais dissipativo, com o valor máximo de 44,8 (no dia 22/03/03), o que demonstra que neste perfil, durante o período de monitoramento, a praia se encontrava com declividade mais baixa, quando comparada com o perfil Pp2 (Figura 9).

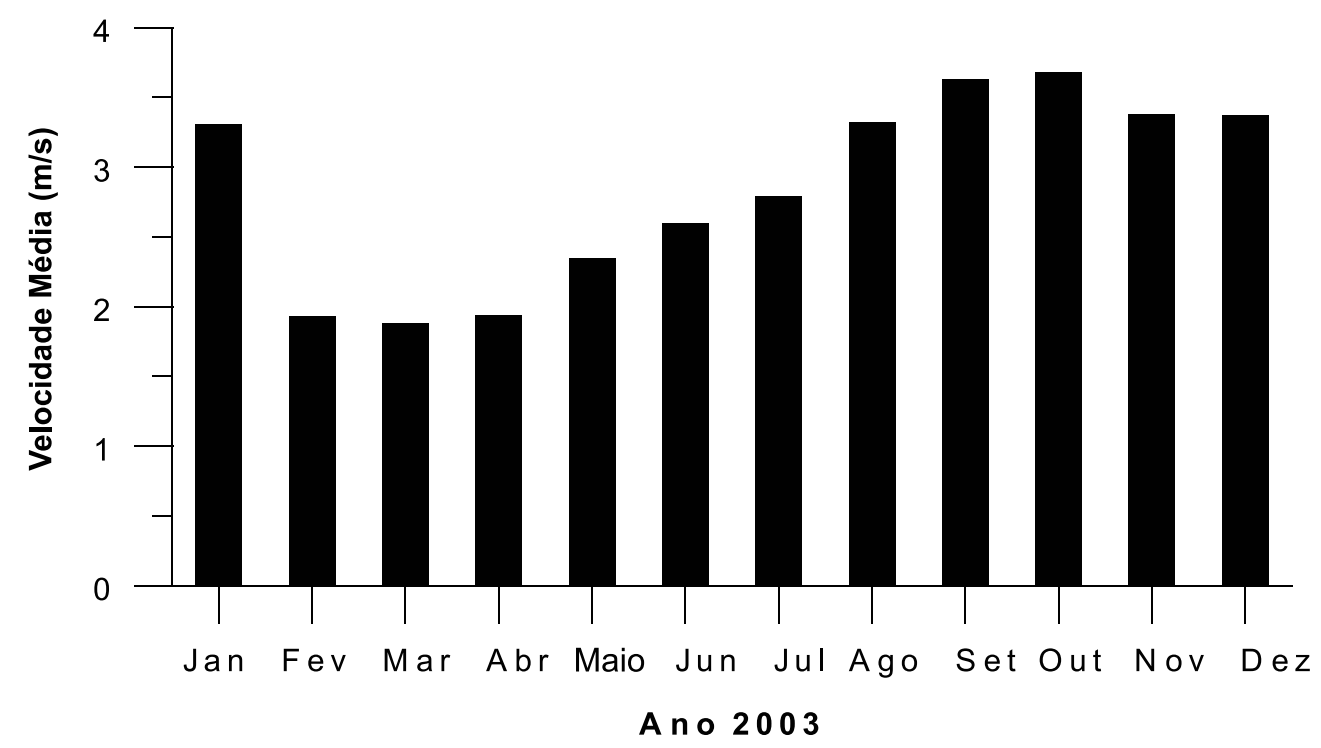

Figura 6 - Intensidade mensal dos ventos (m/s), em Bragança-PA, no ano de 2003

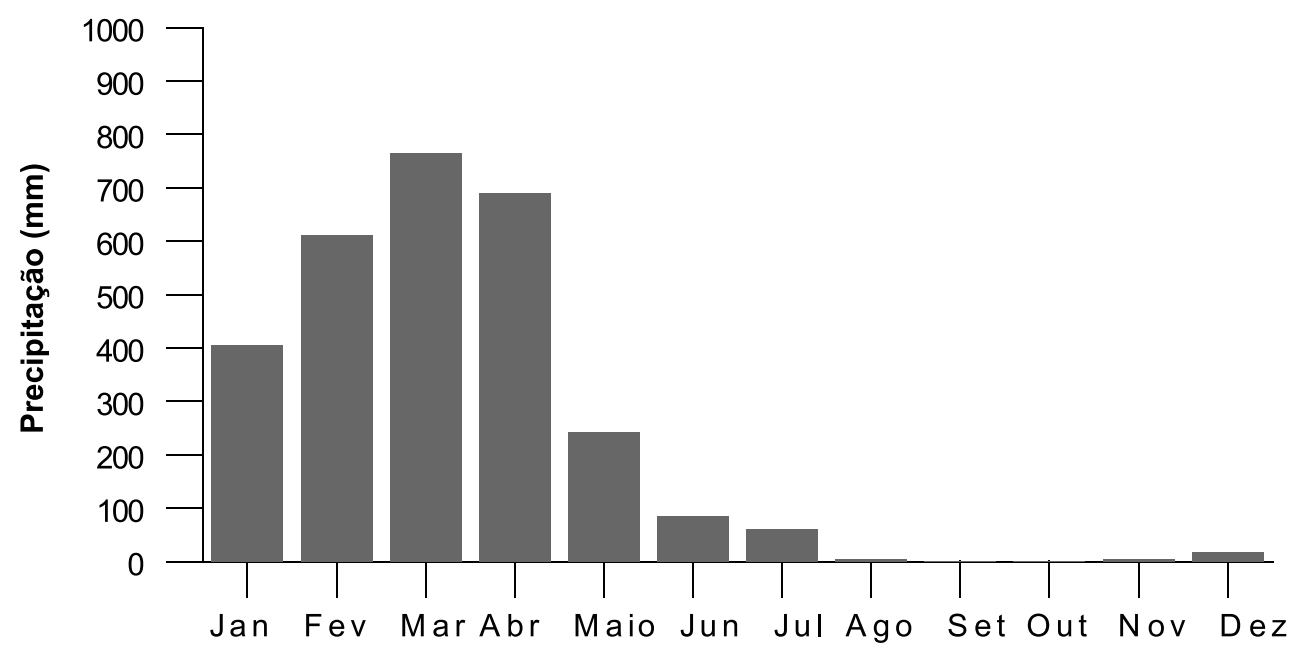

Ano 2003

Figura 7 - Taxa de precipitação (mm) mensal, em Bragança-PA, no ano de 2003 
Short em 2003 comenta que as praias dissipativas ocorrem em áreas de sedimentos finos expostas a grandes ondas tipo swell, de curto período e com rebentação do tipo deslizante. Estas características estão presentes na praia de Ajuruteua tanto em relação à declividade e ao tipo de rebentação, quanto em relação ao tipo de cobertura sedimentar, uma vez que a mesma é formada predominantemente por areia fina (Alves 2001).
Por outro lado, apesar do Pp2 ter apresentado características dissipativas, quanto à definição de Guza \& Inman (1975) e Wright \& Short (1984), foi possível observar também, neste setor, características de praia intermediária, uma vez que a zona de rebentação esteve localizada relativamente próxima à linha de praia, devido à ausência do sistema barra-calha.

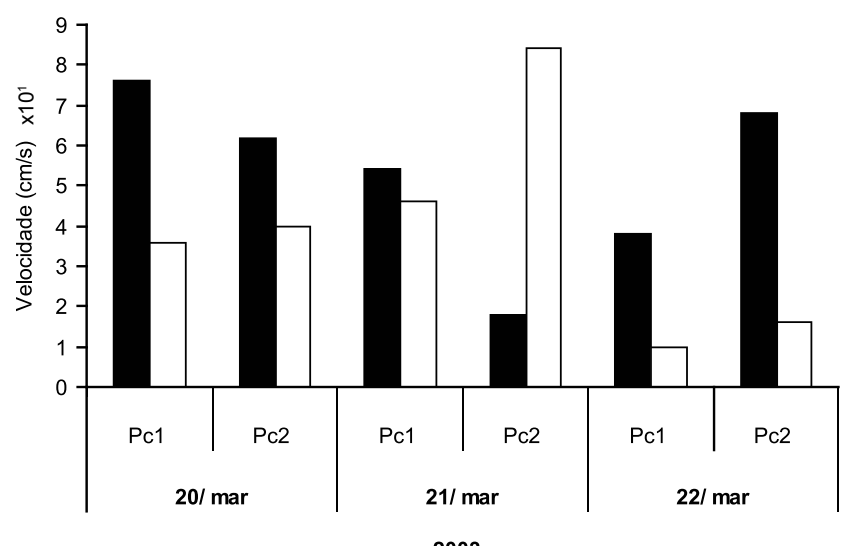

Figura 8 - Velocidade das correntes na zona de $\operatorname{surf}\left(\mathrm{cm} . \mathrm{s}^{-1}\right)$, durante os períodos de enchente e vazante, na praia de Ajuruteua-PA, durante uma maré equinocial de sizígia (março, 2003)

\section{Coeficiente de Rebentação}

Através da aplicação do coeficiente de rebentação $(\beta)$, as ondas na praia em estudo são do tipo deslizante, comum em praias de baixo gradiente, visto que todos os valores registrados foram inferiores a 0,068 , fato comprovado pelo estado morfodinâmico $€>20$.

\section{CONCLUSÃO}

O evento de macromaré equinocial de sizígia constitui um fator preponderante na modificação da configuração morfológica da praia de Ajuruteua, refletindo consideráveis transformações morfodinâmicas de curto período que corresponderam a dados quantitativos (erosão/acresção) dos subambientes praiais. Do ponto de vista hidrodinâmico, a praia de Ajuruteua encontra-se submetida a um regime de macromaré semi-diurna, apresentando dois setores com características morfodinâmicas distintas: o setor SE, com baixa a moderada energia, apresentou ampla zona de rebentação e ondas do tipo deslizante que dissipam a maior parte de sua energia antes de atingir as dunas (dunas preservadas e edificações protegidas); e o setor NW, com moderada a alta energia, apresentou ausência do sistema barra-calha atribuindo a este setor características de praia intermediária, com zona de rebentação localizada próximo à linha de praia (dunas escarpadas e edificações destruídas). Por fim, é importante considerar que a ocupação territorial na zona de dunas contribui para acelerar o curso natural do processo erosivo no sistema praial em questão. 


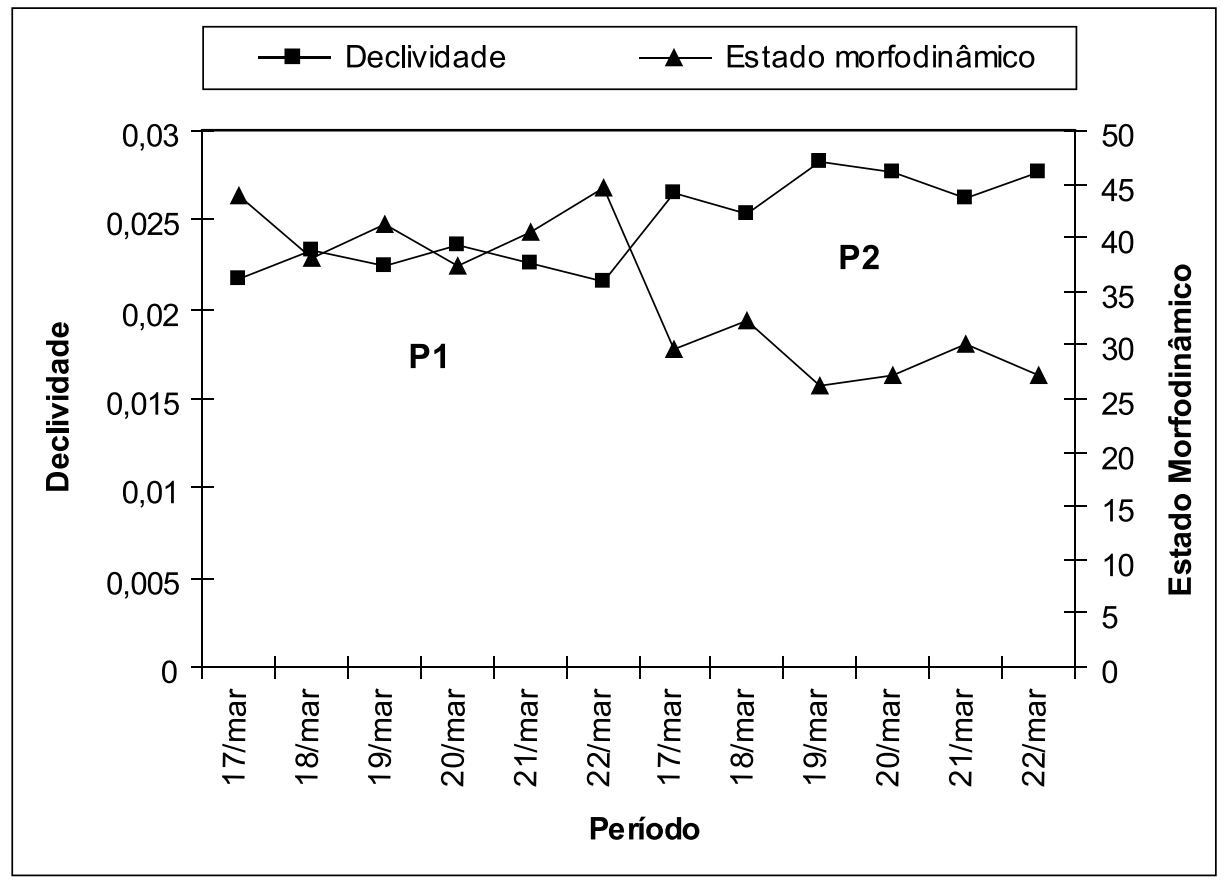

Figura 9 - Relação entre declividade $(\mathrm{m})$ e estado morfodinâmico $(€)$ da praia de Ajuruteua-PA, durante uma maré equinocial de sizígia (março, 2003)

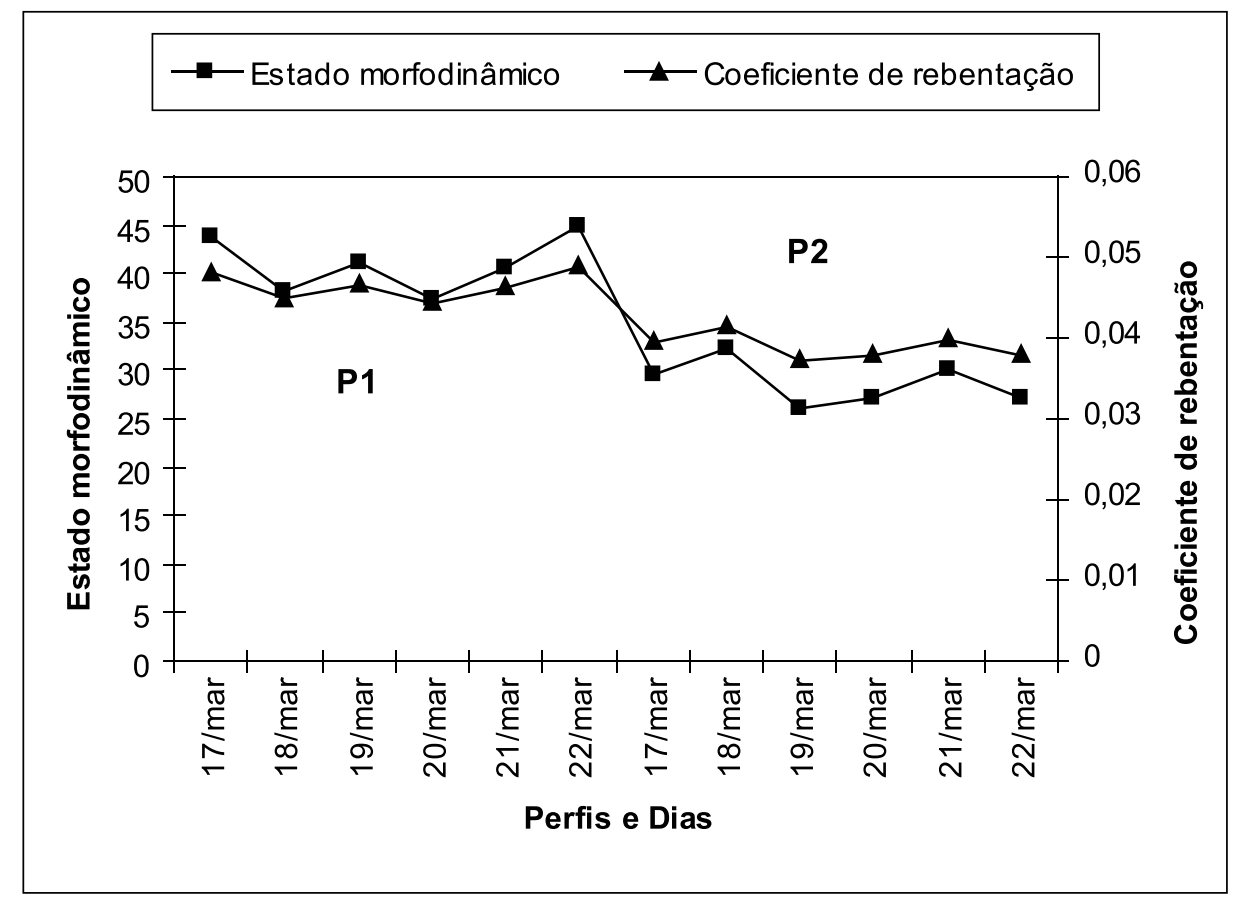

Figura 10 - Relação entre estado morfodinâmico e o coeficiente de rebentação da praia de Ajuruteua-PA, durante uma maré equinocial de sizígia (março, 2003) 
BARBOSA, V. M. et al. Estudo morfodinâmico durante uma maré equinocial de Sizígia ...

\section{AGRADECIMENTOS}

Este estudo é resultado do Projeto Milênio RECOS financiado pelo PADCT e CNPq. Os autores agradecem a Universidade Federal do Pará pela infra estrutura e aos revisores pelas críticas e sugestões ao manuscrito.

\section{REFERÊNCIAS}

ALVES, M.A.M.S. 2001. Morfodinâmica e Sedimentologia da Praia de Ajuruteua - NE do Pará. Belém: Centro de Geociências. Universidade Federal do Pará. Dissertação de Mestrado.

ANFUSO, G. \& GRACIA, F.J. 2005. Morphodynamic Characteristics and Short-Term Evolution of a Coastal Sector in SW Spain: Implications for Coastal Erosion Management. Journal of Coastal Research, 21 (6): 1139-53.

BASCOM, W.N. 1964. Waves and beaches. Doubleday, New York, 268p.

BIRKEMEIER, W.A. 1981. Fast, Accurate Two-Person Beach Survey. Coastal Engineering Technical Aid 81-11. U.S Army Engineer Waterways Experiment Station. Coastal Engineering Research Center, Vicksburg, Mississippi, $22 \mathrm{p}$.

BURAK, S. \& DOGAN, E. \& GAZIOGLU, C. 2004. Impact of urbanization and tourism on coastal environment. Ocean \& Coastal Management, 47 (9-10): 515-527.

DHN. 2003. Tabua das marés para 2003. Fundeadouro de Salinópolis (Estado do Pará). Disponível em: http: www.mar. mil.br/ dhn/tabuas. Acesso em: 10/03/03.

GALVIN, C.J. 1968. Breaker type classification on three laboratory beaches. Journal of Geophysical Research, 73: 3651-3659.

GRUNNET, N.M. \& RUESSINK B.G. \& WALSTRAD.J.R. 2005. The influence of tides, winds and waves on the redistribution of nourished sediment at Terschelling. The Netherlands. Coastal Engineering, 52 (7): 617-631.

GUZA, R.T. \& INMAN, D.L. 1975. Edge waves and beach cusps. Journal of Geophysical Research, 80: 2997-3012.

HORIKAWA, K. 1988. Nearshore Dynamics and Coastal Processes. 1st ed. University of Tokyo Press, $522 \mathrm{p}$.

INMAM, D. L \& FILLOUX, J. 1960. Beach cycles related to tide and local Wind wave regime. Journal of Geology, 68 (2): 225-231.

JACKSON, D.W.T., COOPER, J.A.G. \& RIO, L. DEL. 2005. Geological control of beach morphodynamic state. Marine Geology, 216 (4): 297-314.

KJERFVE, B. \& PERILLO, G. M. E. \& GARDNER, L. R. \& RINE, J. M. \& DIAS, G. T. M. \& MOCHEL, F. R. 2002. Morphodynamics of muddy environments along the Atlantic coasts of North and South America. In: HEALY, T.R. \& WANG, Y. \& HEALY, J-A. (Eds.), Muddy coasts of the world: processes, deposits and functions. Amsterdam, Elsevier Science, 479-532.
KRAUSE, G. \& GLASER, M. 2003. Co-evolving geomorphical and socio-economic dynamics in a coastal fishing village of the Bragança region (Pará, North Brazil). Ocean \& Coastal Management, 46: 859-874.

KRAUSE, G. \& GLASER, M. \& SOARES, C. \& TORRES, D. \& BLANDTT L. \& CUNHA, F.D. 2000. Coastal Dynamics and Socio-Economic Implications. In: Proceedings of the German-Brazilian Workshop on Neotropical Ecosystems, p. 997-1000.

LAFON, V. \& DUPUIS, H. \& BUTEL, R. \& CASTELLE, B. \& MICHEL, D. \& HOWA, H. \& MELO APOLUCENO, D. DE. 2005. Morphodynamics of nearshore rhythmic sandbars in a mixedenergy environment (SW France): 2. Physical forcing analysis. Estuarine, Coastal and Shelf Science, 65 (3): 449-462.

LARA, R.J. 2003. Amazonian mangroves - a multidisciplinary case study in Pará State, North Brazil: introduction. Wetlands Ecology and Management, 11: 217-221.

MAIA, L.P. FREIRE, G.S.S. \& LACERDA, L.D. 2005. Accelerated Dune Migration and Aeolian Transport During EI Nino Events along the NE Brazilian Coast. Journal of Coastal Research, 21 (6): 1121-1126.

MARTORANO, L.G., PEREIRA, L.C., CEZAR, E.G.M. \& PEREIRA, I.C.B. 1993. Estudos climatológicos do Estado do Pará, classificação climática (Köppen) e deficiência hídrica (Thornthwhite, Mather). Belém, SUDAM/EMBRPA, SNLCS, $53 \mathrm{p}$.

MASSELINK, G., KROON, V \& DAVIDSON-ARNOTT, R.G.D. 2006. Morphodynamics of intertidal bars in wave-dominated coastal settings - A review. Geomorphology, 73 (1-2): 3349.

MICALLEF, A. \& WILLIAMS, A.T. 2002. Theoretical strategy considerations for beach management. Ocean \& Coastal Management, 45: 261-275.

NELSON, C. MORGAN, R. WILLIAMS, A.T. \& WOOD, J. 2000. Beach awards and management. Ocean \& Coastal Management, 43 (1): 87-98

PEREIRA, L. C.C, JIMÉNEZ, J.A., MEDEIROS, C. \& COSTA, R.M. 2003. The influence of the environmental status of Casa Caiada and Rio Doce beaches (NE-Brazil) on beaches users. Ocean \& Coastal Management, 46 (11-12): 1011-1030.

PEREIRA, L.C.C., RIBEIRO, M. de J.S., GUIMARÃES, D. de O. \& COSTA, R.M. da. 2006. Formas de uso e ocupação na praia de Ajuruteua-PA (Brasil). Desenvolvimento e Meio Ambiente, 13: 19-30.

SHORT, A.D. 1991. Macro-meso tidal beach morphodynamics - an overview. Journal of Coastal Research, 7 (2): 417-436.

SHORT, A.D. 1999. Handbook of beach and shoreface morphodynamics, Jonh Wiley and Sons, Chichester, 379p.

SHORT, A.D. 2003. Australia beach systems - The morphodynamics of wave through tide-dominated beachdune systems. IN: Proceedings of the Brazilian Symposium on Sandy Beaches: Mophodynamics, Ecology, Uses, Hazards and Management. Journal of Coastal Research, 35: 7-20. 
BARBOSA, V. M. et al. Estudo morfodinâmico durante uma maré equinocial de Sizígia ...

SHORT, A.D.1982. Morphodynamics of a macrotidal beach. Marine Gelogy, 50: 97-128.

SILVA, M. G. L. 2001. Avaliação multitemporal da dinâmica costeira da praia do pescador, Bragança (Norte do Brasil). Belém: Universidade Federal do Pará. Centro de Geociências. Dissertação de Mestrado.

SOUZA FILHO, P W.M. \& PARADELLA, W.R. 2003 Use of synthetic aperture radar for recognition of coastal geomorphological features, land use assessment and shoreline changes in Bragança coast, Pará, Northern Brazil. Annals of the Brazilian Academy of Science, 75: 341-56.

SOUZA FILHO, P. W. M., MARTINS, E. do S.F. \& COSTA, F. R. 2006. Using mangroves as a geological indicator of coastal changes in the Bragança macrotidal flat, Brazilian Amazon: a remote sensing data approach. Ocean \& Coastal Management, 49: $462-475$

SOUZA FILHO, P.W.M \& EL ROBRINI, M. 1998. As Variações de Nível do mar e a Estratigrafia de Seqüências da Planície Costeira Bragantina, Nordeste do Pará (Brasil). Boletim do Museu Paraense Emilio Goeldi, Ciências da Terra, 10: 1-34.

SOUZA FILHO, P.W.M \& EL-ROBRINI, M. 2000. Coastal zone Geormorphology of the Bragança área, northeast of amazon region, Brazil. Revista Brasileira de Geociências, 30: 518-522.
SOUZA FILHO, P.W.M \& EL-ROBRINI, M. 1997. A Morfologia, Processos de Sedimentação e Litofácies dos Ambientes Morfossedimentares da Planície Costeira Bragantina-Nordeste do Estado do Pará (Brasil). Geonomos, 4 (2): 1-16.

SOUZA FILHO, P.W.M. \& PARADELLA, W.R. 2002. Recognition of the main geobotanical features along the Bragança mangrove coast (Brazilian Amazon Region) from Landsat TM and Radarsat-1 data. Wetlands Ecology and Management, 10: 123-132p.

SOUZA-FILHO, P.W.M., TOZZI, H.A. de M. \& El-ROBRINI, M. 2003.Geomorphology, Land-use and Environmental hazards in Ajuruteua Macrotidal Sandy beach, Northern Brazil. IN: Proceedings of the Brazilian Symposium on Sandy Beaches: Mophodynamics, Ecology, Uses, Hazards and Management. Journal of Coastal Research, 35: 580-589.

WRIGHT, L. D. \& SHORT, A.D. 1984. Morphodynamics variability of surf zones and beach: a synthesis. Marine Geology, 50: 97-128. 